\title{
ELENCO DEGLI AUTORI
}

Maria Patrizia Bologna

Università degli Studi di Milano

maria.bologna@unimi.it

Silvia Dal Negro

Libera Università di Bolzano

Silvia.DalNegro@unibz.it

José Luis García Ramón

Università Cattolica del Sacro Cuore di Milano

jose.luis.garcia.ramon@gmail.com

Jacopo Garzonio

Università degli Studi di Padova

jacopo.garzonio@unipd.it

Giovanni Gobber

Università Cattolica del Sacro Cuore di Milano

giovanni.gobber@unicatt.it

Diego Pescarini

CNRS, Université Côte d'Azur, BCL

diego.pescarini@univ-cotedazur.fr

Domenica Romagno

Università di Pisa

domenica.romagno@unipi.it

Giancarlo Schirru

Università degli Studi di Napoli L'Orientale

gschirru@unior.it 
Massimo Vai

Università degli Studi di Milano

massimo.vai@unimi.it

Mariarosaria Zinzi

Università degli Studi di Firenze

mariarosaria.zinzi@unifi.it 